\title{
Slow pathway modification for atrioventricular node re-entrant tachycardia: fast junctional tachycardia predicts adverse prognosis
}

\author{
K J Lipscomb, A M Zaidi, A P Fitzpatrick
}

\begin{abstract}
Objective-To examine the cycle length of the junctional tachycardia often seen during successful slow pathway ablation for atrioventricular (AV) node re-entrant tachycardia, to determine whether shorter cycle lengths predict imminent atrioventricular block.

Design-Retrospective analysis of consecutive patients undergoing slow pathway modification. Intracardiac recordings were analysed after digital storage to determine the development of junctional tachycardia, its duration and maximum, minimum, and mean cycle length, occurrence of heart block, persistent slow pathway conduction, or later confirmed recurrence of AV node re-entrant tachycardia.

Setting-Regional cardiac centre.

Patients-136 consecutive patients undergoing electrophysiological study found to have typical "slow-fast" AV node re-entrant tachycardia and subject to 137 slow pathway modification procedures.

Results-During successful temperature feedback controlled radiofrequency energy application, junctional tachycardia developed in 133 of 137 procedures. During ablation, 10 patients had evidence of AV block (first degree in seven patients and third degree in three), and 17 others had retrograde junctional atrial (JA) block. In these 27 patients, the junctional tachycardia was rapid, with a minimum (SD) cycle length 291 (47) ms. Conduction recovered quickly in all but two patients, one of whom required permanent pacing. Junctional tachycardia with normal AV and JA conduction in the other 111 patients was of a significantly slower minimum cycle length (537 (123) $\mathrm{ms} ; \mathrm{p}<0.0001$ ).

Conclusions-Fast junctional tachycardia with cycle lengths under $350 \mathrm{~ms}$ seen during slow pathway modification is a predictor of conduction block, suggesting proximity to the compact node. Radiofrequency energy application should be terminated immediately to prevent development of AV block. An "auto cut off” facility for cycle lengths shorter than $350 \mathrm{~ms}$ could be built into radiofrequency ablation systems to increase safety.

(Heart 2001;85:44-47)
\end{abstract}

Keywords: junctional tachycardia; atrioventricular node re-entrant tachycardia; slow pathway modification

Manchester Heart Centre, University Department of Cardiology, Manchester Royal Infirmary, Oxford Road, Manchester M13 9WL, UK

K J Lipscomb

A M Zaidi

A P Fitzpatrick

Correspondence to: Dr Fitzpatrick

adam.fitzpatrick@man.ac.uk

Accepted 4 September 2000
An anatomical approach to slow pathway modification for atrioventricular node re-entry tachycardia is an established method of treatment with a high success rate. ${ }^{1-4}$ Atrioventricular (AV) block requiring permanent pacing may occur in as many as $2.0 \%$ of cases. ${ }^{145}$ During the application of radiofrequency energy, junctional tachycardia is often seen and is thought to be a marker of thermal injury and successful slow pathway ablation. ${ }^{6-8}$ It has been suggested that fast junctional ectopy with a short cycle length and associated ventriculoatrial block may herald imminent AV block. ${ }^{6} 79$

In this study we sought to evaluate the frequency and characteristics of junctional tachycardia observed during slow pathway modification with a view to providing guidelines for reducing patient risk from AV block.

\section{Methods}

Between January 1996 and February 2000, 136 consecutive patients with typical "slowfast" AV node re-entrant tachycardia underwent slow pathway modification at a regional cardiac centre. All antiarrhythmic agents were discontinued at least five half lives before electrophysiological study. Patients were sedated with midazolam and diamorphine. Three 6 French quadripolar electrodes were inserted through the right femoral vein and positioned in the high right atrium, the apex of the right ventricle, and across the tricuspid valve to record the His potential. A 7 French luminal decapolar electrode was positioned in the coronary sinus through the right internal jugular vein.

A baseline electrophysiological study, using isoprenaline if necessary, was performed to 
confirm the diagnosis of typical AV node re-entrant tachycardia. Baseline measurements of atrium to His bundle (AH) and His bundle to ventricle (HV) intervals were obtained. Slow pathway modification was then performed using an anatomically guided approach with biplane digital fluoroscopy. A mid-septal position between the $\mathrm{His}$ and coronary sinus electrodes was used to target ablation, together with an atrial to ventricular ratio of approximately $1: 5$ on the bipolar electrogram recorded from the distal electrode of the rove catheter, not dependent upon the recording of a slow pathway potential.

Radiofrequency energy was delivered using an EPT Blazer 7 French, $4 \mathrm{~mm}$ tip, thermistor equipped, steerable ablation catheter and an EPT RX 2000 temperature feedback controlled generator (EPT, Sunnyvale, California, USA). A target temperature of $55^{\circ} \mathrm{C}$ and maximum energy of $55 \mathrm{~W}$ was selected in all cases, and radiofrequency energy delivered for a preset maximum of 60 seconds. A stable catheter position during radiofrequency energy application was confirmed by fluoroscopy. Continuous intracardiac recordings were stored digitally on a Prucka Cardiolab system (Prucka Engineering, Austin, Texas, USA) and analysed retrospectively. For each patient, during the application of radiofrequency energy the development of junctional tachycardia was noted, together with the number of beats of junctional tachycardia, and the maximum, minimum, and mean cycle length. Junctional tachycardia was defined by a cycle length $<600 \mathrm{~ms}$, the absence of normal of AV conduction from a preceding $\mathrm{P}$ wave, "low to high" atrial activation (atrial electrograms in the His recording catheter preceding atrial electrograms in the high right atrial catheter), and a change in cycle length or activation pattern from the re-entrant tachycardia. Loss of 1:1 ventriculoatrial (VA) relation or development of first, second, or third degree AV block was recorded, together with the presence of persistent slow pathway conduction at the end of the procedure and any later confirmed recurrence of AV node re-entrant tachycardia. The number of energy applications was recorded.

After radiofrequency application, atrial overdrive and extrastimulus pacing were repeated to refractoriness, both with and without the intravenous infusion of isoprenaline at rates of 1-3 $\mu \mathrm{g} /$ minute, raising the baseline heart rate by at least $30 \%$. If tachycardia could still be induced, further energy was delivered and testing repeated. Patients were monitored by ECG telemetry overnight following the procedure and a further 12 lead ECG obtained before discharge.

STATISTICAL ANALYSIS

The mean junctional tachycardia cycle length during radiofrequency application in patients who developed AV block was compared with the mean junctional tachycardia cycle length in patients without block using an unpaired $t$ test. Values are given as mean (SD).

\section{Results}

Data from 136 consecutive patients undergoing 137 slow pathway modification procedures were studied. The study group contained 38 male and 98 female patients with a mean age of 45.3 (16.0) years. One patient underwent two ablation procedures separated by an interval of three months after the confirmed recurrence of AV node re-entrant tachycardia.

A successful initial outcome was obtained in all 137 procedures, with no inducible AV node re-entrant tachycardia with and without isoprenaline infusion at the end of the procedure. Persistent slow pathway conduction with single AV nodal echoes was seen in 10 individuals. Radiofrequency energy application was accompanied by development of junctional tachycardia in 133 of the 137 procedures.

In 10 patients radiofrequency application was followed by AV block. This was transient first degree block in six, transient third degree block in two, persistent first degree block at 24 hours in one individual with continuing evidence of slow pathway conduction, and persistent third degree block in one patient who required permanent pacing. One patient who developed transient first degree AV block showed junctional atrial (JA) block before cessation of radiofrequency energy application. Seventeen other patients had evidence of prolonged JA conduction during junctional tachycardia and then transient 2:1 JA block. The minimum cycle length observed in this group of 27 patients with AV or JA block was 291 (47) ms, with a mean cycle length of the junctional tachycardia of 426 (92) $\mathrm{ms}$. The mean number of beats of continuous junctional tachycardia before termination of radiofrequency energy was 13 (9).

The junctional tachycardia observed in the remaining 106 patients during successful radiofrequency application, accompanied by and followed by normal AV conduction, was of significantly longer cycle length than the junctional tachycardia resulting in JA or AV block. The minimum cycle length was 431 (111) ms and the mean cycle length was 537 (123) ms $(p<0.0001)$.

There were no significant differences in baseline electrocardiographic or electrophysiological characteristics between the patients who developed transient AV block and those who did not. The number of energy applications in the group that developed AV or JA block (2.4 (1.9)) was significantly less than in the patients who did not (3.3 (2.4); $\mathrm{p}<0.001)$. Cases of AV block were no more likely to occur during the second or subsequent energy applications than during the first application.

The junctional tachycardia cycle length observed in 10 patients with persistent slow pathway conduction was 445 (124) ms. None of the patients with persistent slow pathway conduction had a recurrence during a mean follow up of 28 (10) months.

There were two confirmed recurrences of AV node re-entrant tachycardia. One individual who developed fast junctional tachycardia 
(minimum cycle length $<300 \mathrm{~ms}$, mean cycle length $345 \mathrm{~ms}$ ) during radiofrequency application, but without evidence of AV block, had a recurrence at six weeks and was successfully ablated at the second attempt. A second patient had a recurrence of clinical tachycardia at 24 hours after developing sustained (37 beats) slow junctional tachycardia (mean cycle length $704 \mathrm{~ms}$ ) during radiofrequency energy, and having no evidence of inducible tachycardia or slow pathway conduction at the end of the procedure. This patient declined a further ablation procedure.

\section{Discussion}

Slow pathway modification using an anatomically guided approach is now established as an effective method of treating atrioventricular node re-entry tachycardia. ${ }^{1-4}$ Previously, fast pathway ablation has been described but slow pathway modification is now the preferred approach because of the lower incidence of $\mathrm{AV}$ block. ${ }^{1351011}$ Delivery of radiofrequency energy during AV nodal, fast and slow pathway ablation has been associated with the development of accelerated junctional tachycardia..$^{6-8}$ 12-14 The precise mechanism of the junctional tachycardia remains unclear but it is likely to be the effect of enhanced automaticity in AV nodal tissue with thermal injury, and the occurrence of such junctional tachycardia is associated with successful elimination of AV nodal re-entrant tachycardia. ${ }^{6}{ }^{14}$

There is a clear but small risk of AV block with slow pathway modification and it has been suggested that junctional tachycardia with a shorter cycle length may predict impending AV block. ${ }^{79}{ }^{15}$ This finding was not confirmed by Jentzer and colleagues in a study of 52 patients undergoing slow pathway modification. ${ }^{6}$ It has been suggested that the appearance of VA block during junctional ectopy heralds the onset of AV block. ${ }^{6}$

This large single centre study confirms that the development of junctional tachycardia during radiofrequency energy application is associated with a high success rate. It also confirms observations found elsewhere in a smaller group of patients ${ }^{7}$ that shorter junctional tachycardia cycle lengths are associated with increasing risk of transient and permanent AV block. It is difficult to define a precise cut off junctional rate indicating risk with the small number of adverse events seen in this study, but junctional tachycardia rates of \pm 1 SD indicate that a cycle length of less than $350 \mathrm{~ms}$ appears to predict reversible and permanent block. The clinical implications of this observation are that a continuous beat to beat digital display of cycle length during radiofrequency energy application may aid rapid recognition of critical tachycardia cycle lengths under $350 \mathrm{~ms}$, enabling prompt termination of radiofrequency energy to prevent the development of AV block. Although loss of 1:1 VA relation during ablation may be more easily recognised, this study illustrates that only one of 10 patients with subsequent AV block had JA block before cessation of energy application. An optional automated cut off of radiofrequency energy delivery linked to hazardous cycle length shortening might facilitate safe delivery of energy for slow pathway modification in $\mathrm{AV}$ node re-entrant tachycardia.

This study also supports the observation that after successful radiofrequency ablation of the slow pathway in patients with AV node re-entrant tachycardia, residual slow pathway conduction does not correlate with clinical tachycardia recurrence. ${ }^{13}$ In 10 of 137 procedures, residual slow pathway function was demonstrated but was not associated with clinical recurrence over a mean follow up period of 28 (10) months.

\section{CONCLUSIONS}

The observation of junctional tachycardia during radiofrequency ablation of the slow pathway is a useful predictor of successful cure of AV node re-entrant tachycardia, but short tachycardia cycle lengths under $350 \mathrm{~ms}$ are associated with an increased risk of transient and permanent AV block which may not always be heralded by AV dissociation during ablation. Rapid termination of energy delivery is required to avoid the need for permanent pacing, and might be made available as an "auto cut off” system within radiofrequency generator systems.

1 Kay GN, Epstein AE, Dailey SM, et al. Selective radiofrequency ablation of the slow pathway for the treatment of atrioventricular nodal reentrant tachycardia. evidence for involvement of perinodal myocardium within the reentrant circuit. Circulation 1992;85:167588 .

2 Wathen $\mathrm{M}$, Natale A, Wolfe K, et al. An anatomically guided approach to atrioventricular node slow pathway ablation. Am F Cardiol. 1992;70:886-9.

3 Jazayeri MR, Hempe SL, Sra JS, et al. Selective transcatheter ablation of the fast and slow pathways using radiofrequency energy in patients with atrioventricular nodal reentrant energy in patients with atrioventricular
tachycardia. Circulation 1992;85:1318-28.

tachycardia. Circulation 1992;85:1318-28.
4 Jackman WM, Beckman KH, McCelland JH, et al. Treatment of supraventricular tachycardia due to atrioventricular node reentry by radiofrequency ablation of the slow tricular node reentry by radiofrequency ab
pathway. $N$ Engl f Med 1992;327:313-18.

5 Hindricks G. Incidence of complete atrioventricular block following attempted radiofrequency catheter modification of the atrioventricular node in 880 patients. Results of the Multicentre European Radiofrequency Survey (MERFS), the working group on arrhythmias of the European Society of Cardiology. Eur Heart f 1996;17:82-8.

6 Jentzer JH, Goyal R, Williamson BD, et al. Analysis of junctional ectopy during radiofrequency ablation of the slow pathway in patients with atrioventricular nodal reentrant tachycardia. Circulation 1994;90:2820-6.

7 Thakur RK, Klein GJ, Yee R, et al. Junctional tachycardia: a useful marker during radiofrequency ablation for atrioventricular node reentrant tachycardia. $\mathcal{F} \mathrm{Am}$ Coll Cardiol 1993;22:1706-10.

8 Boyle NG, Anselme F, Monahan K, et al. Origin of junctional rhythm during radiofrequency ablation of atrioventricular nodal reentrant tachycardia in patients

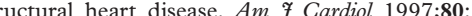
$575-80$

9 Schumacher B, Tebbenjohanns J, Pfeiffer D, et al. Junctional arrhythmias in radiofrequency modification of the atrioventricular node. $Z$ Kardiol 1995;84:977-85.

10 Jazayeri MR, Akhtar M. Electrophysiological behaviour of the atrioventricular node after selective fast or slow pathway ablation in patients with atrioventricular nodal reentrant tachycardia. PACE 1993;16:623-8.

11 Prystowsky EN. Atrioventricular node reentry: physiology and radiofrequency ablation. PACE 1997;20:55271 .

12 Yeung-Lai-Wah JA, Alison J, Lonergan L, et al. High success rate of atrioventricular node ablation with radiofrequency energy. $\mathcal{F}$ Am Coll Cardiol 1991;18:1753-8.

13 Manolis AS, Wang PJ, Estes M. Radiofrequency ablation of slow pathway in patients with atrioventricular nodal reentrant tachycardia. Do arrhythmia recurrences correlate with persistent slow pathway conduction or site of successful ablation? Circulation 1994;90:281519. 
14 Epstein MR, Saul JP, Fishberger SB, et al. Spontaneous accelerated junctional rhythm: an unusual but useful observation prior to radiofrequency catheter ablation for observation prior to radiofrequency catheter ablation for
atrioventricular node reentrant tachycardia in young patients. PACE 1997;20:1654-61.
15 Chen SA, Chiang CE, Tai CT, et al. Transient complete atrioventricular block during radiofrequency blation of slow pathway for atrioventricular nodal reentrant tachycardia. Am f Cardiol 1996;77:136770 .

\section{Commentary}

Many theoretical aspects of atrioventricular (AV) node structure and function remain controversial, but in the last 10 years, clinical cardiac electrophysiologists have developed a pragmatic approach to radiofrequency catheter ablation of the AV node slow pathway, which is now among the safest and most successful of all forms of invasive treatment for heart disease.

Atrioventricular nodal re-entrant tachycardia (AVNRT) accounts for about $70 \%$ cases of paroxysmal supraventricular tachycardia. The re-entry circuit is formed by two separate electrical pathways in the region of the AV node named "fast" and "slow" according to the relative conduction velocities. The usual position of these pathways is shown in fig 1. Unlike accessory atrioventricular pathways, these pathways are not histologically discrete structures. They are two (or sometimes more) functionally separate routes by which the cardiac impulse may travel through a zone of myocardium containing sheets of transitional cells with properties that are intermediate between those of atrial myocardium and the compact $\mathrm{AV}$ node.

Under resting conditions, AV conduction is over the fast pathway. Typically, AVNRT occurs owing to a re-entry circuit within the AV node with anterograde conduction down the slow pathway and retrograde conduction back up the fast pathway (fig 1C). AVNRT can be prevented by interruption of the circuit either by ablation of the slow pathway, fast pathway, or compact $\mathrm{AV}$ node. Ablation of the compact $\mathrm{AV}$ node results in complete heart block, requiring permanent pacing. Ablation of the fast pathway, which is only a few millimetres in length and is directly adjacent to the compact AV node, carries a 5-20\% risk of damage to the compact AV node and complete heart block. In contrast, the slow pathway is $1-2 \mathrm{~cm}$ in length, and the proximal end can be ablated with a low risk of injury to the compact AV node, thus preserving normal AV conduction.

Most operators use an anatomical approach to identify the slow pathway; the key to which is identification in the right anterior oblique projection of the region of the atrial septum called the triangle of Koch (fig 1A). The apex of the triangle is identified by the His bundle recording catheter, the base by the coronary sinus recoding catheter, and the right hand side by the tricuspid annulus. Radiofrequency lesions are created at one or more sites in the triangle of Koch, starting at the base (posterior) and working towards the apex (anterior). The hallmark of AV nodal or transitional cell tissue is a flurry of ectopic junctional rhythm during radiofrequency energy delivery. If junctional rhythm is seen during a test lesion, energy delivery may be continued for a minute or more. Signs of impending complete heart block during slow pathway ablation will prompt the operator to halt energy delivery immediately. These include prolongation of the $\mathrm{AH}$ interval, or the presence of any junctional rhythm which is not conducted retrogradely as well as anterogradely. To these signs, Lipscomb and colleagues have now added the emergence of rapid junctional rhythm with a cycle length of $350 \mathrm{~ms}$ or less, corresponding to a heart rate of greater than 170 beats per minute. Careful attention to these features may minimise the risk of the subsequent development of complete heart block. Even when ablating in apparently safe positions away from the His recording catheter, great care is required since there is significant variability in the anatomy of the slow and fast pathways. After ablation, success is confirmed by the absence of inducible AVNRT during programmed stimulation.
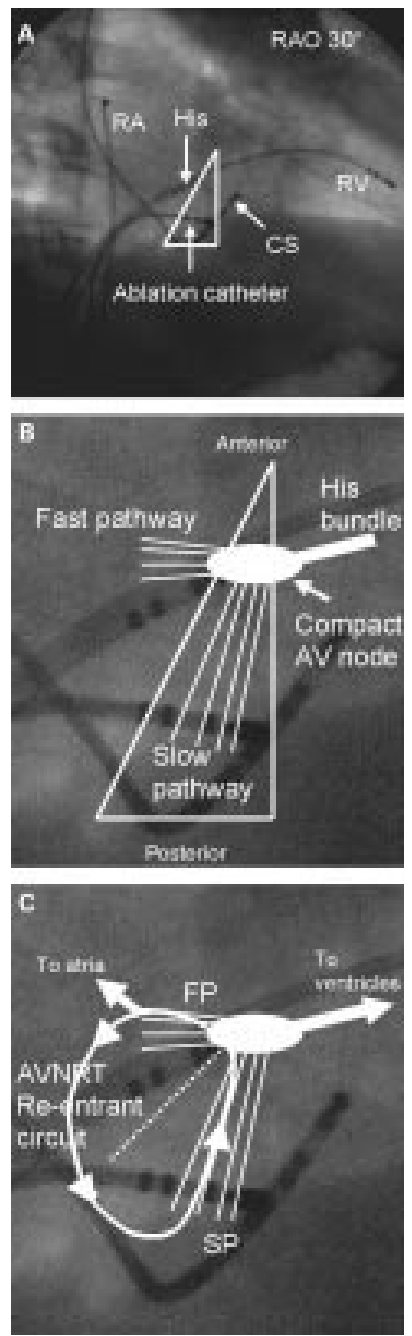

Figure 1 Position of the "fast" and "slow" electrical pathways in the region of the $A V$ node. $C S$, coronary sinus catheter; FP, fast pathway;
right atrial catheter; $R A O$, right right atrial catheter; $R A O$, right
anterior oblique projection; $R V$ right ventricular catheter; SP, slow pathway. 